\title{
Self-Complementary Metasurface for Designing Narrow Band Pass/Stop Filters
}

\author{
J. D. Ortiz, J. D. Baena, Member, IEEE, V. Losada, F. Medina, Fellow, IEEE, R. Marqués, Member, IEEE, and \\ J. L. Araque Quijano
}

\begin{abstract}
A self-complementary metasurface is studied in this paper. The metasurface is a 2-D periodical arrangement of unit cells formed by a metallic printed split ring resonator and its complementary counterpart. It is demonstrated that this structure behaves like a very selective band-pass filter for a certain linear polarization while band-stop filtering is achieved for the orthogonal polarization over the same frequency range. This idea opens the door to a new class of frequency selective surfaces made of connected and unconnected elements, whose filtering properties are mechanically tunable from band-pass to band-stop by rotating the surface or the polarization.
\end{abstract}

Index Terms-Babinet's principle, frequency selective surface (FSS), self-complementary metasurface.

\section{INTRODUCTION}

$\mathbf{F}$ REQUENCY selective surfaces (FSS) made of connected metal elements behave as band-pass filters, while unconnected metal elements yield band-stop behavior [1], [2]. Split ring resonators (SRRs) [3] and other related resonators used in bulk metamaterials have been incorporated into FSS design. The newly created devices are often called metasurfaces [4]. The distinctive characteristic of metasurfaces is the small electrical size of their unit cells. Secondary grating lobes are then avoided, even when the metal pattern is not printed on a high dielectric constant substrate. Moreover, electrically small unit cells affords the engineering of effective homogeneous surface impedances [4]. Recently, an interesting self-complementary FSS (which remains unaltered when air and metal are interchanged) has been proposed [5]. This structure works as a polarizer around some specific frequency in the mm-wave range. The structure in [5] is not a metasurface because the

Manuscript received February 01, 2013; revised March 21, 2013; accepted March 30, 2013. Date of publication April 25, 2013; date of current version June 03, 2013. This work has been supported by the Colombian Administrative Departament of Science, Technology and Innovation (COLCIENCIAS, project 1101-521-29389) and Spanish Ministerio de Ciencia e Innovación (project Consolider EMET CSD2008-00066 and TEC2010-16948).

J. D. Ortiz and J. D. Baena are with the Department of Physics, Universidad Nacional de Colombia, Bogotá, Colombia (e-mail: jdortizc@unal.edu.co; jdbaenad@unal.edu.co).

V. Losada is with the Department of Applied Physics I, Universidad de Sevilla, Sevilla, Spain (e-mail: losada@us.es).

F. Medina and R. Marqués are with the Department of Electronics and Electromagnetism, Universidad de Sevilla, Sevilla, Spain (e-mail: medina@us.es; marques@us.es).

J. L. Araque Quijano is with the Electric and Electronics Engineering Department, Universidad Nacional de Colombia, Bogotá, Colombia (e-mail: jlaraqueq@unal.edu.co).

Color versions of one or more of the figures in this paper are available online at http://ieeexplore.ieee.org.

Digital Object Identifier 10.1109/LMWC.2013.2258001 unit cell is comparable to the resonance wavelength (this fact has implications in terms of grating lobes). In spite of its reasonably good behavior, that structure provides relatively low frequency selectivity and an unsymmetrical pass/stopband, which might be undesirable features if a highly selective band-pass/band-stop filter is the goal. A high selectivity structure, based on a small self-complementary unit cell, with good band symmetry was theoretically demonstrated in [6]. However, the FSS in [6] makes use of a large number of identical lumped circuit elements. These components should be free from parasitic effects above $2 \mathrm{GHz}$, which is rather unrealistic, making difficult the practical implementation of the idea in [6]. In this paper we propose a more viable and fully planar version of the same idea. It is experimentally demonstrated that this self-complementary metasurface can be used as band-pass or band-stop filter depending on the polarization state of the incident radiation.

\section{THEORY}

The proposed self-complementary metasurface is shown in Fig. 1. Although this structure may recall the proposal in [7], where two complementary and cascaded metal sheets were used, the structure in Fig. 1 can be studied by using the Babinet's principle (a single metal sheet is used). Let us first consider normal incidence. In the long wavelength limit, the central strips of the SRR behave like a capacitor that can be electrically driven by a $x$-polarized tangential electric field. The capacitance can be approximated as $C=e C_{\text {pul }}$, where $e$ is the length of the capacitor and $C_{\mathrm{pul}}$ the capacitance per unit of length of the coplanar strips. Following [8] we can write:

$$
C_{\mathrm{pul}}=\epsilon_{0} \frac{K^{\prime}(k)}{K(k)}+\frac{\epsilon_{0}\left(\epsilon_{r}-1\right)}{2} \frac{K^{\prime}\left(k_{1}\right)}{K\left(k_{1}\right)}
$$

where $K(k)$ is the complete elliptic integral of the first kind, $K^{\prime}(k)=K\left(k^{\prime}\right), k=g /(g+2 w), k^{\prime 2}=1-k^{2}$, and $k_{1}=$ $\sinh (\pi g /(4 h))[\sinh (\pi(g+2 w) /(4 h))]^{-1}$.

Furthermore, the external U-shaped strip behaves as a selfinductance. The inductance $L$ can be approximated from the inductance of an infinite metal strip carrying a uniform current $([9$, p. 322])

$$
L=\frac{\mu_{0} l}{2 \pi}\left[\log \left(\frac{2 l}{w}\right)+\frac{1}{2}\right]
$$

where $w$ is the strip width and $l$ can be approximated as $l=$ $2 c+2 d-g-4 w$. Therefore, the SRR can be approximated as an 


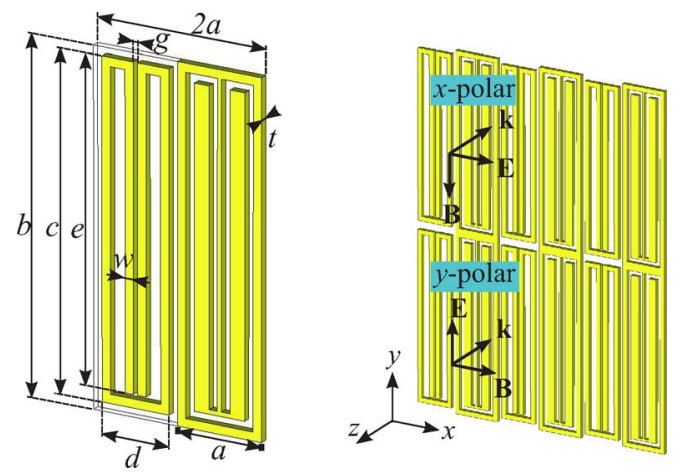

Fig. 1. On the left side, the unit cell consisting of one SRR and one C-SRR with the same dimensions. On the right side, a view of the self-complementary metasurface iluminated by $x$ - or $y$-polarized plane waves.

LC circuit resonating at the frequency $f_{0}=1 /[2 \pi \sqrt{L C}]$. According to the Babinet's principle, the C-SRR should exhibit a strong response to an $x$-directed magnetic field ( $y$-polarization) at the same resonance frequency of the SRR (zero thickness perfect conductors are assumed). Far away from the resonance frequency, the region filled by SRRs should be almost transparent, while the region with C-SRRs should behave like continuous metallic strips without slots. The metasurface should then behave like a strip grating. For wavelengths much larger than the period, the strip grating behaves as a polarizer thus providing the correct baselines for band-pass and band-stop filtering. These bands appear near the resonance frequency, $f_{0}$, due to the excitation of the SRRs and C-SRRs. For $x$-polarization the wave could go through the effective strip grating but, in the vicinity of $f_{0}$, the impinging wave is strongly scattered by SRRs and a stopband should appear. This band will become a passband if the polarization state is rotated $90^{\circ}$ as a consequence of self-complementarity and Babinet's principle.

\section{NuMERICAL SimULATIONS}

Numerical simulations have been carried out with CST Microwave Studio to confirm the reasonings above. The geometrical parameters are the ones in the caption of Fig. 2. Perfect electric conductor (PEC) and zero metal thickness $(t=0)$ have been assumed. The magnitude of the transmission coefficients are shown in Fig. 2. The behavior discussed in the previous paragraph is clearly shown for $x$ - and $y$-polarizations, although certain numerical inaccuracy associated with finite mesh size prevents perfect coincidence of peak/dip frequencies. The resonances were found at $2.18 \mathrm{GHz}$ while the theoretical expressions (1) and (2) predict $2.33 \mathrm{GHz}$. Note that the frequency selectivity and symmetry of the bands are higher than in [5]. The half-power bandwidth of our structure is $1.4 \%$ while more than $50 \%$ bandwidth can be estimated from Fig. 1(a) in [5]. Moreover, the $-3 \mathrm{~dB}$ points are located at $-0.78 \%$ and $+0.64 \%$ of $f_{0}$ in our case, while for the structure in [5] these figures are $-37 \%$ and $+18 \%$.

In order to demonstrate that the stopband is mainly controlled by the SRRs and the passband by the C-SRRs, we have depicted the surface electric currents at resonance in the insets of Fig. 2. Only the SRRs are excited for $x$-polarization, while only the C-SRRs are excited for $y$-polarization. A different way to

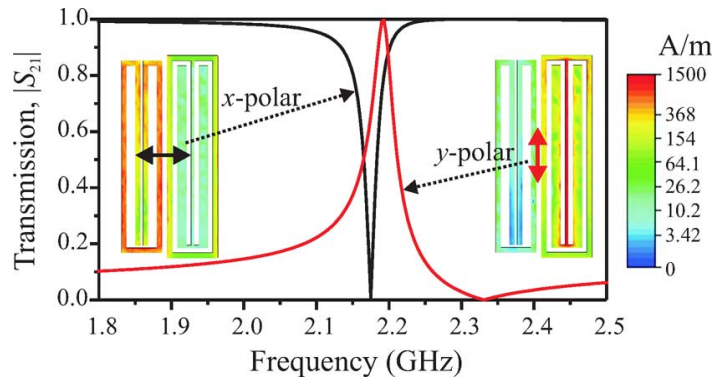

Fig. 2. Simulated transmission through the self-complementary metasurface made of PEC. Following the notation presented in Fig. 1, the geometrical parameters are (all in $\mathrm{mm}$ ): $a=4, b=16, c=15.2, d=3.2, g=0.2, e=$ $14.6, w=0.4$, and $t=0$. The insets represents surface electric currents at $2.18 \mathrm{GHz}$.

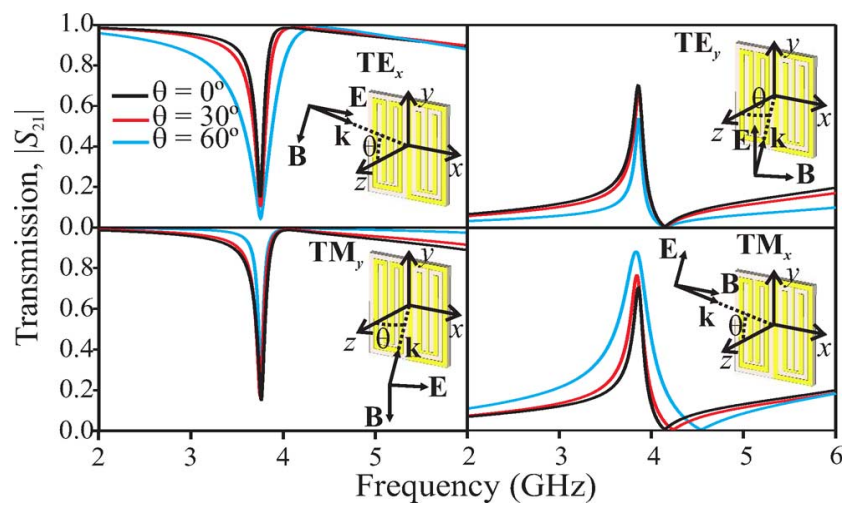

Fig. 3. Simulated transmission through the self-complementary metasurface made of copper on a dielectric substrate. Plots for angles of incidence $0^{\circ}$ (black), $30^{\circ}$ (red), and $60^{\circ}$ (cyan), and for canonical polarizations $\mathrm{TE}_{x}, \mathrm{TM}_{y}, \mathrm{TE}_{y}$, and $\mathrm{TM}_{x}$. Following the notation in Fig. 1, the geometrical parameters are (all in $\mathrm{mm}): a=3.4, b=8, c=7.2, d=2.6, e=0.2, l=6.6, w=0.4$, and $t=0.035$. A dielectric substrate was used with thickness $h=0.5 \mathrm{~mm}$ and $\epsilon_{r}=2.5(1-j 0.0075)$.

verify the independence between the sub-arrays is to remove one type of resonator while its complementary counterpart is kept in place. We have observed that when the C-SRRs are removed (filling the slots with metal), the stopband for the $x$-polarization remains unchanged while the passband for $y$-polarization disappears. Conversely, when the SRRs are removed $y$-polarization is not affected while the stopband for $x$-polarization is lost.

In practice, some lossy conductor on some dielectric substrate must be used. In order to investigate the effects of ohmic losses and the dielectric layer, a realistic structure made of copper on a conventional microwave substrate has been simulated. Fig. 3 shows the transmission coefficient of the structure under $x$-polarization illumination $\left(\mathrm{TE}_{x}\right.$ or $\mathrm{TM}_{y}$ for $\left.0^{\circ}\right)$ and $y$-polarization $\left(\mathrm{TE}_{y}\right.$ or $\mathrm{TM}_{x}$ for $\left.0^{\circ}\right)$. The previously reported behavior is still observed for copper on dielectric: a sharp stopband for $x$-polarization and a dual passband for $y$-polarization. Expressions (1) and (2) lead to a resonance frequency of $3.77 \mathrm{GHz}$, in good agreement with simulations. Ohmic losses, of course, preclude perfect reflection or perfect transmission. Even though one could expect some frequency shift between the peak and the dip due to the presence of the dielectric slab and the finite thickness of the metal sheet, the observed shift is very small because the two causes for shifting partially compensate each other. 

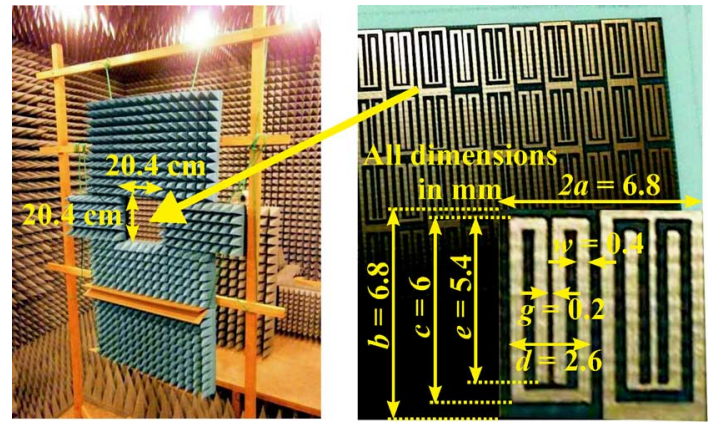

Fig. 4. Experimental setup. The sample is placed at the hole in the absorber wall. Dimensions of the sample: $20.4 \mathrm{~cm} \times 20.4 \mathrm{~cm}$. Two horn antennas are placed in front and back of the FSS at a distance of $185 \mathrm{~cm}$. Copper thickness $t=$ $0.035 \mathrm{~mm}$, dielectric thickness $h=0.49 \mathrm{~mm}$, and $\epsilon_{r}=2.43(1-j 0.0018)$.

In the design of a FSS the angular and polarization dependences are key features and they require some attention. Usually, high stability in the response is desired because the surface could receive radiation from any direction of the space and with any polarization state. Fig. 3 also shows the transmission coefficient under oblique incidence for angles $30^{\circ}$ and $60^{\circ}$. Each of the four graphics in Fig. 3 is focused on one of the canonical linear polarization states $\left(\mathrm{TE}_{x}, \mathrm{TM}_{y}, \mathrm{TE}_{y}\right.$, and $\left.\mathrm{TM}_{x}\right) . \mathrm{TE}_{x}$ and $\mathrm{TM}_{y}$ polarizations yield stopbands, as expected, because the ordinary SRRs are mainly excited by the incident $E_{x}$ (see left column of Fig. 3). Conversely, $\mathrm{TE}_{y}$ and $\mathrm{TM}_{x}$ excitations yield passbands (see right column of Fig. 3). Note that the angular response is quantitatively stable within $30^{\circ}$, but only qualitatively stable for incident angles within $60^{\circ}$. The resonance frequency remains stable but the bandwidth and the height/depth at the peak/dip location is variable for large incident angles. This is due to the collaboration of orthogonal flux of magnetic or electric field on the SRR or the C-SRR (flux depends on the angle of incidence).

\section{EXPERIMENT}

A first proof of concept experiment has been carried out. A square $20.4 \mathrm{~cm} \times 20.4 \mathrm{~cm}$ sample was fabricated using $A R L O N$ $\mathrm{CuClad} 250 \mathrm{LX}$. The geometrical and physical parameters are included in Fig. 4. The characterization was done in anechoic environment by measuring $S_{21}$ between two horn antennas (suitable for 3.5-5.5 GHz measurements). The sample was placed at the center of the anechoic chamber, $185 \mathrm{~cm}$ far from each antenna, and oriented perpendicularly to the imaginary line joining the antennas. The incident wave was, approximately, a plane wave (angle of incidence is $0^{\circ}$ ). Several pieces of absorbing material were used to minimize diffraction at the borders. Fig. 5 shows the measured $\left|S_{21}\right|$. A resonance appears at $4.59 \mathrm{GHz}$ what is very close to the value $4.58 \mathrm{GHz}$ obtained from (1) and (2). The stopband for $x$-polarization and the passband for $y$-polarization are clearly observed. A reasonably good agreement between experiment (solid lines) and simulation (dashed lines) is observed. However, an important qualitative disagreement is observed below the resonance. It is known ([10, p. 5]) that the finite size of FSSs will lead to standing surface wave effects that show up in frequencies $20 \%-30 \%$ below the resonance when the inter-element spacing is less than half wavelength (as it is

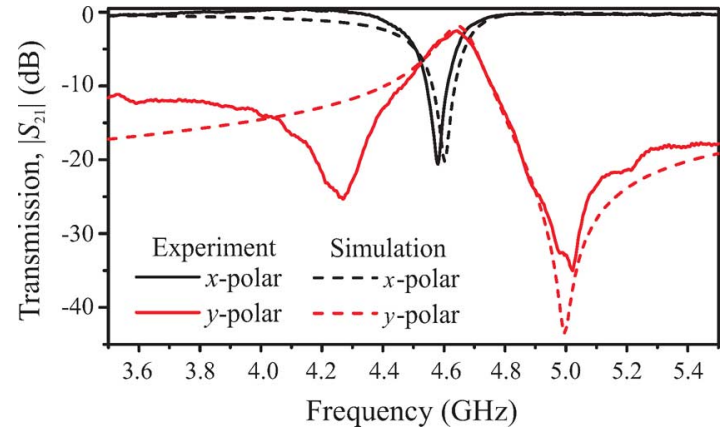

Fig. 5. Measured transmission (solid lines) and simulated transmission (dashed lines) for $x$-polarization (black) and $y$-polarization (red), only under normal incidence. The geometry of the actual sample is specified in Fig. 4. Measured data are normalized by the transmission through the empty hole. Simulated data corresponds to an infinite array.

our case). Therefore, it is the authors belief that the observed disagreement is a finite size effect that might be mitigated by using a larger sample.

\section{CONCLUSION}

The self-complementary metasurface proposed in this letter behaves as a band-pass filter for a given linear polarization and, simultaneously, as a band-stop filter for the orthogonal polarization. The metasurface made with PEC exhibits the expected results. Although the studied sample of lossy metal shows worse transmission spectra, the band-pass and band-stop filtering features still remain for the corresponding polarizations. Besides, we have demonstrated that this behavior is very stable when the incident angle is varied within $60^{\circ}$. This idea could open the door to a new class of frequency selective surfaces made of connected and unconnected elements, whose filtering properties are mechanically tunable from band-pass to band-stop by rotating the surface.

\section{REFERENCES}

[1] T. K. Wu, Frequency Selective Surfaces and Grid Arrays. New York: Wiley, 1995.

[2] B. A. Munk, Frequency Selective Surfaces: Theory and Design. New York: Wiley, 2000.

[3] J. B. Pendry, A. J. Holden, D. J. Robins, and W. J. Stewart, "Magnetism from conductors and enhanced nonlinear phenomena," IEEE Trans. Microw. Theory Tech., vol. 47, no. 11, pp. 2075-2084, Nov. 1999.

[4] C. L. Holloway, E. F. Kuester, J. A. Gordon, J. O?Hara, J. Booth, and D. R. Smith, "An overview of the theory and applications of metasurfaces: The two-dimensional equivalents of metamaterials," IEEE Antennas Propag. Mag., vol. 54, pp. 10-35, Apr. 2012.

[5] M. Beruete, M. Navarro-Cía, I. Campillo, P. Goy, and M. Sorolla, "Quasioptical polarizer based on self-complementary sub-wavelength hole arrays," IEEE Microw. Wireless Compon. Lett., vol. 17, no. 12, pp. 834-836, Dec. 2007.

[6] J. D. Ortiz, J. D. Baena, R. Marqués, and F. Medina, "A band-pass/stop filter made of SRRs and C-SRRs," in Proc. IEEE Int. Symp. Antennas Propag. (APS/URSI), Spokane, WA, 2011, pp. 2669-2672.

[7] X. D. Hu, X. L. Zhou, L. S. Wu, L. Zhou, and W. Y. Yin, "A miniaturized dual-band frequency selective surface (fss) with closed loop and its complementary pattern," IEEE Antennas Wireless Propag. Lett., vol. 8, pp. 1374-1377, 2009.

[8] V. Fouad-Hanna, "Finite boundary corrections to coplanar stripline analysis," Electron. Lett., vol. 16, pp. 604-606, 1980.

[9] E. B. Rosa, "The self and mutual inductances of linear conductors," Bulletin Bur. Standards, vol. 4, pp. 301-344, 1908.

[10] B. A. Munk, Finite Antenna Arrays and FSS. New York: Wiley-IEEE Press, 2003 